\title{
A seasonal pattern in the onset of polymyalgia rheumatica
}

\author{
M A Cimmino, R Caporali, C M Montecucco, S Rovida, E Baratelli, M Broggini
}

Department of

Rheumatology,

M A Cimmino

University Institute of

Medical Pathology,

IRCCS San Matteo,

Pavia

R Caporali

C M Montecucco

Department of

Medical Statistics,

Genoa University

$S$ Rovida F del Ponte Hospital,

Varese

E Baratelli, M Broggini

Correspondence to:

Dr M A Cimmino,

Via Casaregis 2-6,

16129 Genova, Italy.

30 August 1989
Genoa University

Department of Medicine,

Accepted for publication

\begin{abstract}
The seasonal distribution in the onset of polymyalgia rheumatica (PMR) was determined in 58 patients with the disease and compared with that in 44 patients affected by rheumatoid arthritis of elderly onset (EORA). Thirty six (62\%) cases of PMR developed during May to August; by contrast, only 14 (31\%) cases of EORA developed in the same months, this latter disease failing to show any seasonal clustering. The monthly distribution of PMR correlated with outside temperature and hours of sunshine. These data suggest that PMR might be triggered by such factors as actinic damage of superficial vessels or infective agents with a seasonal cycle. Finally, the summer clustering of PMR may be helpful in the differential diagnosis from EORA.
\end{abstract}

Polymyalgia rheumatica (PMR) is characterised by pain and stiffness in the shoulder and pelvic girdles, accompanied by a pronounced acute phase response. Although its cause is unknown, genetic factors may have a role, as suggested by reports of familial clustering ${ }^{1}$ and the association with the HLA antigen DR4. ${ }^{2}$ Hepatitis $B$ virus has occasionally been proposed as a triggering agent, ${ }^{3}$ but this observation has not been confirmed in other series. ${ }^{4}$ As PMR is often heralded by acute and severe symptoms most patients can recall exactly the date of onset. A seasonal clustering of cases of PMR in both summer and winter was described by Mowat and Hazleman ${ }^{5}$ and confirmed by Bird et al. ${ }^{6}$ On the other hand, two other more recent reports failed to show any seasonal trend. ${ }^{78}$

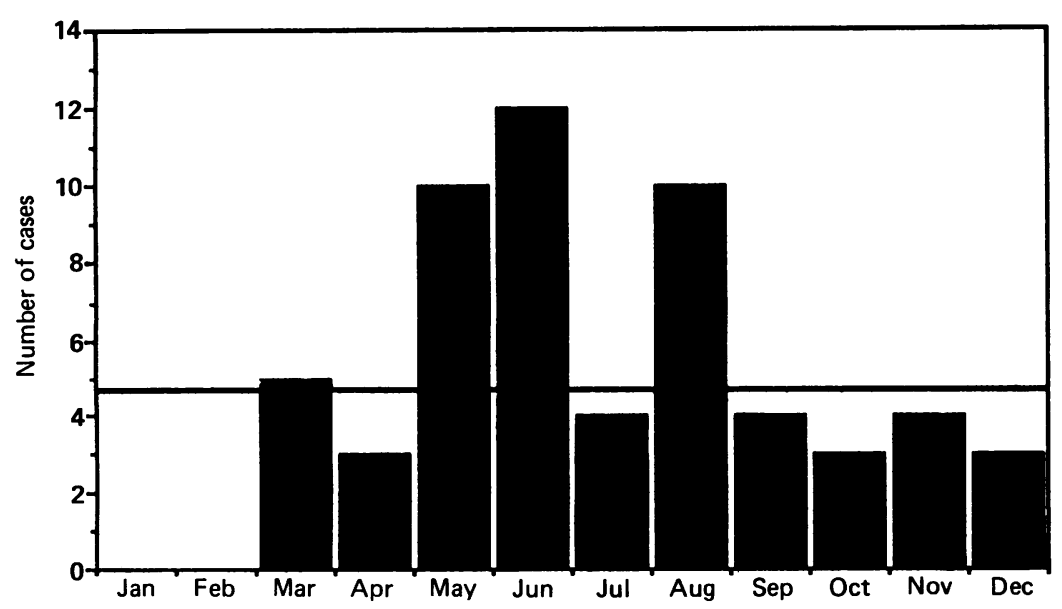

Figure 1: Monthly incidence of onset of symptoms in new cases of polymyalgia rheumatica (cumulative data for 1986-1988). Bar indicates the value expected from the yearly mean.
This study attempted to determine if there is a seasonal distribution for PMR and to ascertain the importance of seasonal variation in discriminating PMR from elderly onset rheumatoid arthritis (EORA).

\section{Patients and methods}

Seventy three patients with PMR were asked if the onset of their disease had been abrupt and if they could remember exactly when symptoms began. PMR was diagnosed according to the criteria of Jones and Hazleman, ${ }^{9}$ modified by the inclusion of only patients over 50 years of age with an erythrocyte sedimentation rate (ESR) of at least $40 \mathrm{~mm} /$ first hour. Fifty eight patients (44 women and 14 men, with a mean age of 69 years, range 53-83 years) could specify the month of onset. Peripheral joint involvement was present in 14 patients and temporal arteritis in 10. All the patients were seen at three rheumatological outpatient clinics in northern Italy. The date of the first visit, the highest ESR, the maximum dose of corticosteroids, the duration of treatment, the presence of fever, and the amount of weight loss, if any, were also noted.

The months of onset were similarly recorded in 80 control patients with EORA. Elderly onset rheumatoid arthritis was defined as rheumatoid arthritis beginning after the age of 65 . In 44 patients the onset of EORA was acute and its date was remembered. Thirty three patients were women and 11 were men; the mean age was 71 years (range 65-92 years). A polymyalgic onset was reported in 15 patients with EORA.

Diagnosis was confirmed by a follow up of at least eight months (mean duration one and a half years). In the patients with PMR the rheumatoid factor was consistently negative and erosive joint damage was not seen.

The mean monthly temperatures and hours of sunshine for 1986-1988 in the areas where the patients lived were obtained from the Chiavari observatory, Chiavari, Genova, and from the Centro Geofisico Prealpino, Varese.

Statistical methods for non-parametric data, including the $\chi^{2}$ test and Spearman's rank order correlation coefficient, were used.

\section{Results}

Fifty eight out of 73 (79\%) patients with PMR were able to recall the month of onset of the disease, which for 29 of them fell in 1988, for 16 in 1987, and for 13 in 1986. New cases of PMR were few in autumn and winter, increasing in spring, and peaking in summer (fig 1). From 
May to August the incidence of PMR was significantly higher than in the other months ( 36 new cases vs $22 ; \chi^{2}=22.49 ; \mathrm{p}<0.0001$ ). No differences were found in this seasonal pattern when the data were broken down by the different rheumatological clinics or by the year of onset (data not shown).

Figure 2 shows the monthly rate of the

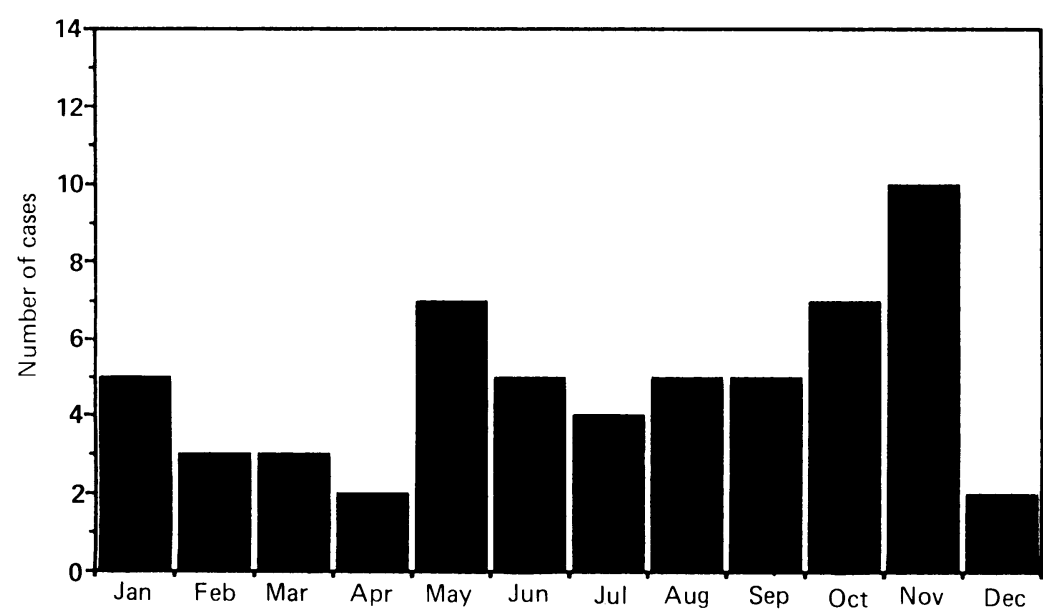

Figure 2: Monthly incidence of first examinations for polymyalgia rheumatica at three Italian clinics.

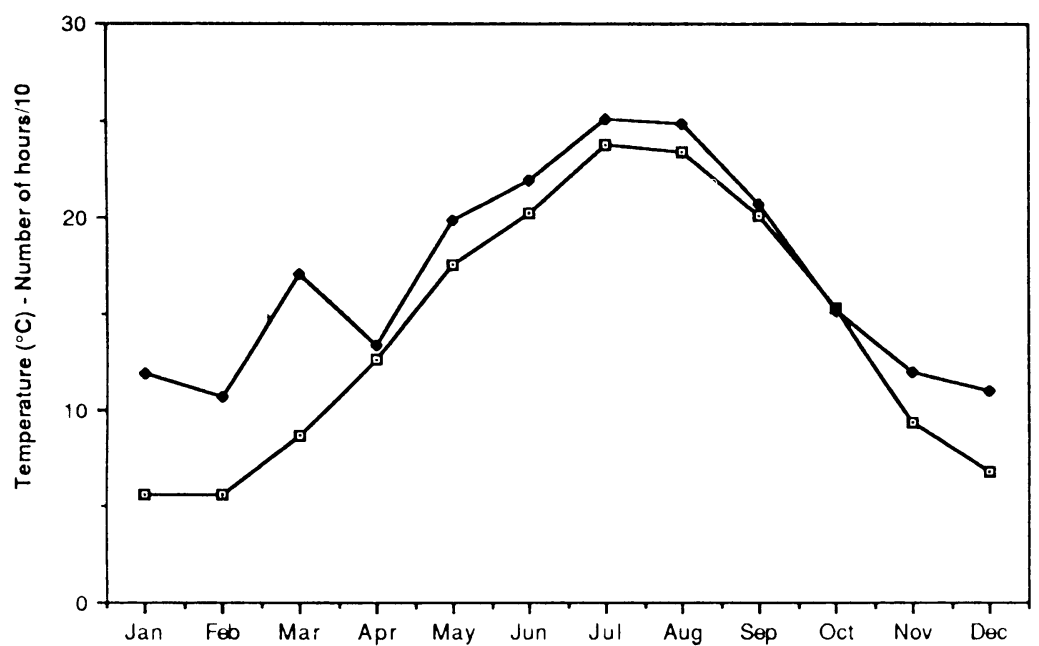

Figure 3: Mean temperatures (-־- ) and hours of sunshine (- - ) by month between 1986 and 1988 .

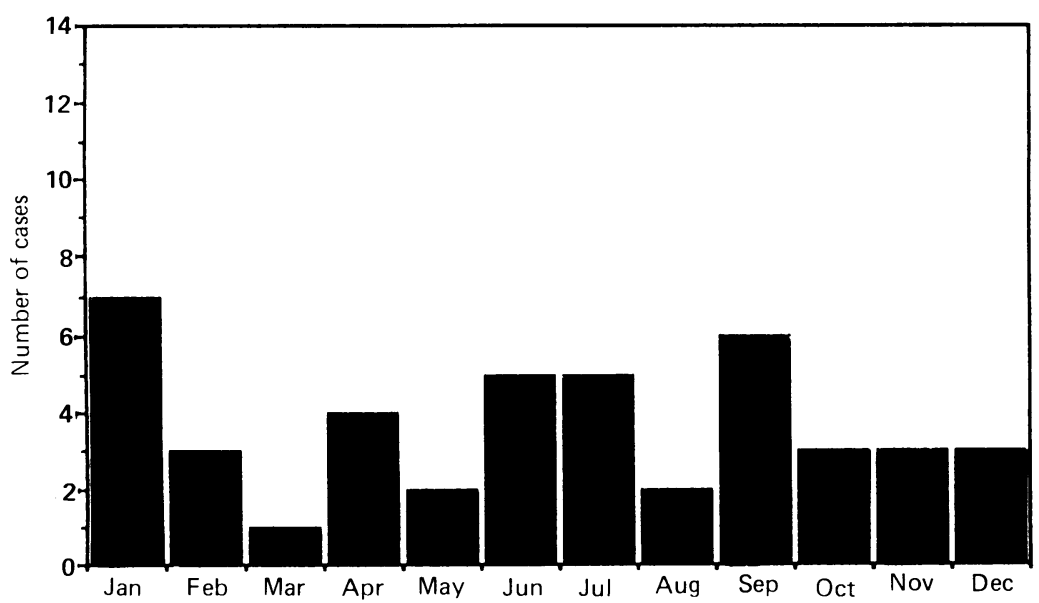

Figure 4: Monthly incidence of onset of symptoms in new cases of elderly onset rheumatoid arthritis (cumulative data for 1986-1988). patients' first visit, which was roughly even throughout the year (between the four month periods January to April, May to August, and September to December $\chi^{2}=3 \cdot 34$; NS). There was a significant difference between the two distributions $(\mathrm{p}<0.02)$, excluding the possible bias of selective referral of this type of patient in a given period of the year.

Figure 3 shows the mean monthly temperatures and hours of sunshine. The mean temperature from May to August was more than twice that during the remaining months. The differences in the number of monthly sunshine hours were also pronounced. The monthly incidence of PMR was significantly correlated with the mean temperatures $(p<0.05)$ and hours of sunshine $(p=0.01)$.

The 36 patients with onset of disease from May to August were compared with the remaining 22 patients in terms of clinical signs and symptoms, treatment, and ESR. No significant differences were found, although the male to female ratio, the mean ESR, and the percentage of patients with fever or weight loss were slightly higher in the 'May to August' group. Conversely, prevalence of temporal arteritis was slightly lower in the patients with summer onset: (five/36; 13.9\%) compared with the remaining patients (five $/ 22 ; 22 \cdot 7 \%$, NS).

Forty four out of $80(55 \%)$ patients with EORA could recall the month of onset of their symptoms. This percentage was significantly lower $\left(\chi^{2}=9 \cdot 2 ; \mathrm{p}<0.01\right)$ than that of the patients with PMR who could do the same $(79 \%)$. The onset of symptoms in 44 patients with EORA, including those with polymyalgia-like attacks, was scattered throughout the year with no seasonal clustering (fig 4). Thirty one per cent of the patients with EORA traced the onset of their symptoms to the four months from May to August compared with $62 \%$ of the patients with PMR $\left(\chi^{2}=7 \cdot 99 \% ; \mathrm{p}<0 \cdot 01\right)$.

\section{Discussion}

This study indicates that there is a seasonal clustering in the summer months of new cases of PMR. A seasonal trend in PMR was originally recognised in Oxford. ${ }^{56}$ It has been denied by Chuang et al in a study of the population in Olmsted County, Minnesota, ${ }^{7}$ and by Coomes et al in London.

Seventy nine per cent of patients with PMR could recall the month of onset of the disease compared with $44 \%$ of patients with EORA, which was related to the PMR usually showing a more abrupt onset. In PMR the incidence of new cases during the warm season from May to August was significantly higher than that in the remaining months $(p<0.0001)$. The flow of new patients was roughly even throughout the year and its slight variations were not considered to bias the sample. In particular, the slight increase in the number of first visits in November may well be related to the mean interval between onset of symptoms and diagnosis $(4 \cdot 3$ months in this series). Moreover, PMR was slightly more severe, and the association of temporal arteritis slightly lower, in the patients with disease which began in the summer. 
A seasonal pattern in the incidence of a disease of unknown origin may be helpful in that it points to agents which usually display seasonal variations. ${ }^{10}$ The summer clustering of new cases of PMR may be related to causes such as higher outside temperatures or increased exposure to sunlight. ${ }^{11}$ Solar radiation, mainly of the infrared type, may disrupt the internal elastic lamina of medium and small sized vessels, inducing the expression of new antigenic determinants. ${ }^{12}$ This vascular damage could be the common pathogenetic mechanism for both the synovitis of PMR and temporal arteritis. The onset of temporal arteritis is characterised by a well defined summer peak, as shown by Kinmont and McCallum. ${ }^{13}$ In our series, however, the number of patients with temporal arteritis was too small to show any seasonal pattern of onset. Alternatively, exposure to sunlight may be a triggering factor by its possible interference with the immunological response. ${ }^{14}$ The OKT8+ suppressor/cytotoxic subset of $\mathrm{T}$ cells, however, increases when healthy volunteers are exposed to artificial actinic radiation in a solarium. ${ }^{14}$ As a result, this feature cannot be considered to be typical of patients with PMR in whom a selective defect of the OKT8+ suppressor/cytotoxic subset of $T$ cells has been described. ${ }^{15} 16$

Exposure to sunlight may be the precipitating agent in PMR, although additional evidence is needed. Two series of data exhibiting seasonality do not necessarily mean that the two phenomena have a cause and effect correlation. None the less, the correlation of the monthly distribution of PMR with hours of sunshine was stronger than that with temperature, suggesting that the first association could probably be more helpful in the search for a cause. The different weather conditions of the regions where previous studies on PMR have been carried out may explain the contrasting results regarding seasonality of onset.

A trivial viral illness, which may trigger an autoimmune process in predisposed elderly subjects, has been reported in many patients with PMR. Data on the prevalence of hepatitis B infection in patients with $\mathrm{PMR},{ }^{3}$ however, have not been confirmed. ${ }^{4} \mathrm{~A}$ recent study has also shown that Epstein-Barr virus is not associated with PMR. ${ }^{17} \mathrm{~A}$ summer peak in the onset of PMR should draw the attention to infections that show the same seasonal trend. In the temperate climate of northern Italy this pattern is seen in infections by such pathogens as enterovirus and Legionella spp, among others. Finally, the observation that IgM antibodies to intermediate filaments are frequently found in the sera of patients with PMR further supports this hypothesis. ${ }^{18}$ In fact, antibodies to intermediate filaments have been reported in patients with viral infections such as infectious mononucleosis, hepatitis $A$, hepatitis $B$, and non-A, non- $B$ hepatitis. These antibodies may be elicited by cross reactivity between viral proteins and intermediate filament proteins.
Seasonality may prove useful in the differential diagnosis between PMR and EORA, which is often difficult in the early stages. In our series the onset occurred from May to August in 62\% of patients with PMR compared with $31 \%$ of patients with EORA $(p<0.01)$. Thus when the standardisation by yearly incidence of the diseases is considered, an inflammatory syndrome affecting mainly the pelvic or shoulder girdles and beginning in the summer months is more likely to be PMR than EORA.

In conclusion, a seasonal onset of PMR has been shown in three different series of outpatients in northern Italy. This may point to a few agents for this disease of unknown origin which share this seasonality. Further studies to examine selectively some behavioural patterns, such as exposure to sunlight and sun sensitising drugs, in patients with PMR could be of interest. Likewise, serological investigations into possible exposure to specific infective agents might also be useful. Professor S Furia, Centro Geofisico Prealpino, and Don G
Leonardini, Osservatorio di Chiavari, provided the climatological Leonardini, Osservatorio di Chiavari, provided the climatological data. Dr C Pesce, National Cancer Institute,
Maryland, USA, provided helpful comments.

1 Kvernebo K, Brath H K. Polymyalgia arteritica. A report on five cases within a family. Scand $\mathcal{F}$ Rheumatol 1980; 9 $187-9$

2 Richardson J E, Gladman D D, Fam A, Keystone E C. HLADR4 in giant cell arteritis: association with polymyalgia rheumatica syndrome. Arthritis Rheum 1987; 30: 1293-7.

3 Bacon P A, Doherty S M, Zuckerman A J. Hepatitis-B antibody in polymyalgia rheumatica. Lancet 1975; ii: 476-8.

4 Bridgeford P H, Lowenstein M, Bocanegra T S, Vasey F B German B F, Espinoza L R. Polymyalgia rheumatica and giant cell arteritis: histocompatibility typing and hepatitis-B
infection studies. Arthritis Rheum 1980; 23: 516-18.

5 Mowat A G, Hazleman B L. Polymyalgia rheumatica: a clinical study with particular reference to arterial disease. f Rheumatol 1974; 1: 190-202

6 Bird H A, Esselinkx W, Dixon A S J, Mowat A G, Wood P H N. An evaluation of criteria for polymyalgia rheumatica. $A n n$ Rheum Dis 1979; 38: 434-9.

7 Chuang T, Hunder G G, Ilstrup D M, Kurland L T. Polymyalgia rheumatica. A 10 -year epidemiologic and clinical study. Ann Intern Med 1982; 97: 672-80.

8 Coomes E N, Ellis R M, Kay A G. A prospective study of 102 patients with the polymyalgia rheumatica syndrome. patients with the polymyalgia rheumatica synd

9 Jones J G, Hazleman B L. Prognosis and management of polymyalgia rheumatica. Ann Rheum Dis 1981; 40: 1-5.

10 Bowie C, Prothero D. Finding causes of seasonal disease using time series analysis. Int $\mathcal{F}$ Epidemiol 1981; 10: 87-92.

11 O'Brien J P. Vascular accidents after actinic (solar) exposure. An aspect of the temporal arteritis/polymyalgia rheumatica syndrome. Int f Dermatol 1987; 26: 366-70

12 O'Brien J P. A concept of diffuse actinic arteritis. The role of actinic damage to elastin in 'age change' and arteritis of the actinic dam A cory elast in 'age change' and arterisis of the Dermatol 1978; 98: 1-13.

13 Kinmont P D C, McCallum D I. The aetiology, pathology and course of giant-cell arteritis. The possible role of ligh sensitivity. Br $\mathcal{F}$ Dermatol 1965; 77: 193-202.

14 Hersey P, Bradley M, Hasic E, Haran G, Edwards A, McCarthy W H. Immunological effects of solarium exposure. Lancet 1983; ii: 545-8.

15 Benlahrache C, Segond P, Auquier L, Bouvet J P. Decrease of the OKT8 positive $\mathrm{T}$ cell subset in polymyalgia rheum atica. Arthritis Rheum 1983; 26: 1472-80.

16 Chelazzi G, Broggini M. Abnormalities of peripheral blood $T$ lymphocyte subsets in polymyalgia rheumatica. Clin $\operatorname{Exp}$ Rheumatol 1984; 2: 333-6.

17 Buchwald D, Sullivan J L, Leddy S, Komaroff A L. 'Chronic Epstein-Barr virus infection' syndrome and polymyalgia Epstein-Barr virus infection' syndrome and
rheumatica. F Rheumatol 1988; 15: 479-82.

18 Dasgupta B, Duke O, Kyle V, Macfarlane DG, Hazleman B L, Panayi G S. Antibodies to intermediate filaments in polymyalgia rheumatica and giant cell arteritis: a sequential study. Ann Rheum Dis 1987; 46: 746-9. 